\title{
Quantum physics from waves: An analogy-based approach for high school
}

\author{
Marcelo Arlego ${ }^{1}$, María de los Ángeles Fanaro ${ }^{* 0}$, Lorenzo Galante ${ }^{3,4}$ \\ ${ }^{1}$ Consejo Nacional de Investigaciones Científicas y Técnicas, Núcleo de Investigación en Educación en Ciencia y Tecnología, \\ Instituto de Física La Plata, Argentina. \\ ${ }^{2}$ Universidad Nacional del Centro de la Provincia de Buenos Aires, Consejo Nacional de Investigaciones Científicas y \\ Técnicas, Facultad de Ciencias Humanas, Núcleo de Estudios Educacionales y Sociales, Argentina. \\ ${ }^{3}$ Politecnico di Torino, Dipartimento Scienza Applicata e Tecnologia and Teaching and Language Laboratory, Torino, Italia. \\ ${ }^{4}$ Museo Storico di Fisica, Centro Studi e Ricerche Enrico Fermi, Roma, Italia.
}

Received on January 20, 2021. Accepted on March 21, 2021.

\begin{abstract}
In this paper we present a proposal for teaching basic aspects of quantum mechanics in high school. Our proposal is based on a wave approach that makes use of the analogies between wave phenomena at macroscopic scales and phenomena at atomic or subatomic scales. Although the classical and the quantum model are completely different, there is a common underlying wave mathematical layer. This allows to address, via analogies, quantum aspects that are not accessible to direct experience. The quantization of energy levels, the quantum interference and the uncertainty relations are examples of quantum aspects having an analog concept in classical physics wave phenomena. In our approach we break the wave-particle "duality", which is totally counterintuitive. Instead we enhance the underlying wave mathematical structure as a path to reach and explain emerging features of particles and quantum systems in general.
\end{abstract}

Keywords: Quantum; teaching; secondary school; wave model.

\section{Introduction}

Quantum mechanics plays an essential role in today's technology. Without quantum physics, there would be no cell phones or internet, to cite two paradigmatic cases. However, quantum physics is considered an abstruse and complicated subject, since the atomic world is far from all daily experience. For this reason, research in the teaching of quantum mechanics, especially at elementary levels, has been an active and debated field for years, as illustrated in Ref. [1] 6 .

In this work, we propose a strategy that focuses on the underlying wave structure of quantum mechanics as a path to reach and explain corpuscular aspects of quantum systems. The basic idea is to introduce specific parts of wave physics to bring out the typical quantum behaviour of microscopic systems. Traditionally, there is a balance between the wave and particle aspects that, in our opinion, is not totally satisfactory at secondary school level. We rather suggest to introduce an asymmetry: the quantum behaviour arises from the wave model which is the mathematical supporting structure at the base.

From a wave description the quantum behaviour emerges naturally. Just to give some examples:

\footnotetext{
${ }^{*}$ Correspondence email address: mariangelesfanaro@gmail.com
}

1. Spatial localisation arises from the possibility of superposing waves to form a localized wave packet.

2. Quantisation of energy levels emerges from the physics of waves trapped in a finite medium (standing waves).

3. The Uncertainty relations are an intrinsic aspect of waves [7].

However, reversing the direction of the logical sequence is not possible, and therein lie many of the conceptual problems faced when approaching quantum phenomena with a "particle" perspective extrapolated from the macroscopic world. The diffraction of electrons and the discrete energy levels of atoms are examples in which the identification of the electron with a particle is irreconcilable with the experimental evidence. We consider that this is an advantageous approach to understand quantum aspects starting from the inquiry and analysis of everyday wave phenomena.

We promote the use of models and analogy to provide them a useful framework to better understand quantum phenomena. Models and modelling are key tools for scientists, science teachers and science students [8]. They are recognized as mediating instruments between experiment and theory [9, 10. From the educational point of view, models also have a primary role in teaching sciences, if they are mediated by a process of didactic transposition [11]. In addition to the use of models in science 
classes, the didactic use of analogies is recognized, since it represents one of the forms of human reasoning 11,13 . Analogies require correspondence between aspects of the base domain (familiar) and the target domain (unknown) to be established. The process of comparing the aspects that are related is called mapping or transfer. The functions of analogies in teaching are generally explanatory. They are formulated because they help to better understand a phenomenon or problem 14 as well as to formulate questions about the physical interpretation and limits of the adopted model/analogy.

The teaching model with analogies (TWA) developed by Glynn in 1991 provides a general structure for the didactic use of analogies [15]. The TWA usually comprises the following steps to help teachers use analogies systematically and effectively: Introduce the target concept to students; remind students of what they know about the analog (base) concept; identify relevant features of the base concept; connect (map) the similar features of the analog and the target; indicate where the analogy between the analog and the target breaks down and finally draw conclusions. However, here we will not strictly follow these "steps" since we consider it more appropriate to begin with the presentation of phenomena familiar to students, and that these serve as the basis for quantum phenomena, totally new to them. Therefore, we will begin by exploring the classical wave model, and then extrapolate these ideas to quantum phenomena, to establish its fundamental characteristics.

In the next section, we will present the structure of the proposal to teach quantum physics and we will analyze the possible advantages for students to build the quantum concepts. The proposal is organized in four stages, following the natural process of human learning: an interaction between the concepts already acquired and the situations to learn new concepts. The situations are not arbitrary, but carefully selected and created so that students can create the connections necessary for learning these new concepts. Table 1 presents each stage of teaching with its corresponding purpose.

\section{Outline of the Proposal to Teach Quantum Physics}

\subsection{First stage: experiments with mechanical waves}

The first part of the sequence deals with wave phenomenology at the macroscopic level in mechanical systems. Possible low cost experiments are proposed, although eventually, they are also accessible through the web (applets, simulations, videos). The inquiry at this stage is phenomenological and focuses on physical models of mechanical waves. Situations are proposed that students can address through the manipulation of the experiment itself, starting from a simple level of observation and then moving toward relationships among the relevant physical variables.
Table 1: The four planned teaching stages with their objectives.

\begin{tabular}{|l|l|}
\hline Stage & \multicolumn{1}{c|}{ Aim } \\
\hline $\begin{array}{l}\text { 1-Experiments with } \\
\text { mechanical waves }\end{array}$ & $\begin{array}{l}\text { Present wave phenomenology at } \\
\text { the macroscopic level in } \\
\text { mechanical systems to note the } \\
\text { quantization and the } \\
\text { interference. }\end{array}$ \\
\hline $\begin{array}{l}\text { 2-Second Stage: Basic } \\
\text { models to describe } \\
\text { wave phenomena }\end{array}$ & $\begin{array}{l}\text { Quantisation and Interference } \\
\text { models as a bridge between } \\
\text { classical and quantum physics }\end{array}$ \\
\hline $\begin{array}{l}\text { 3-Third Atomic-scale } \\
\text { phenomena }\end{array}$ & $\begin{array}{l}\text { Introduce students of what they } \\
\text { know about the analog (base) } \\
\text { concept and identify relevant } \\
\text { features of the base concept }\end{array}$ \\
\hline $\begin{array}{l}\text { 4-Fourth Stage. Basic } \\
\text { aspects of wave } \\
\text { quantum mechanics }\end{array}$ & $\begin{array}{l}\text { Connect (map) the similar } \\
\text { features of the analog and the } \\
\text { target; indicate where the } \\
\text { analogy between the analog and } \\
\text { the target breaks down; and } \\
\text { draw conclusions }\end{array}$ \\
\hline
\end{tabular}

The experiments begin with a simple pendulum or a mass-spring system, to introduce the phenomenon of oscillations. Students inquire on the meaning of oscillations over time about an equilibrium point. At this stage propagation in space is absent. Once oscillations over time are defined from an experimental and visual perspective, their propagation in space can be addressed. This is a crucial step in which the relation between frequency and wavelength $\lambda$ represent the focal point. This knowledge is required to address the key-topic of interference. In Figure 1 some of the most representative experiments related to the propagation of waves in mechanical media are illustrated.

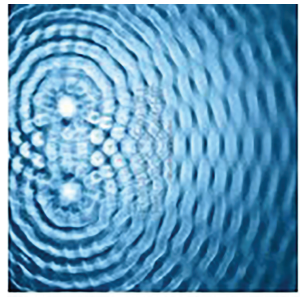

(a)

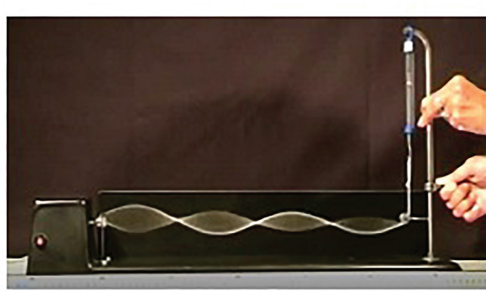

(b)

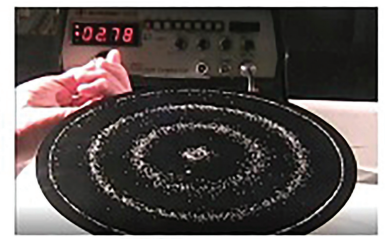

(c)

Figure 1: Proposed experiments illustrating mechanical phenomena associated with waves. (a) mechanical wave interference in a bucket of water. (b) Generation of standing waves in one (a) and two dimensions (c). 
The wave interference phenomenon is shown by the example of water waves in a bucket in Fig. 1(a). The brightest points in this figure indicate the position of the stirrers, which generate circular waves propagating from that position. The waves coming from both sources interact generating a pattern of bright and dark regions (right in the figure). Another typical wave phenomenon is the formation of standing waves in a finite medium. This pattern is characterized by the emergence of nodes, which are points or regions where there is no motion. A typical standing wave experiment is shown in Figure 1 (b) in which a string fixed at one end (the right in this case) and attached to a rotor at the other is capable of supporting one-dimensional standing waves in the string. In this device, the speed of the rotor, as well as the tension of the string are variable parameters, which allow generating a variety of waves with different numbers of nodes (oscillation modes) and amplitudes (wave height) depending on these parameters. The same phenomenology of standing waves, but in two dimensions can be analyzed with a system as depicted in Fig. 11(c), which consists of a metal plate attached to a speaker, which is fed with a variable sound frequency generator. By placing sand arbitrarily on the speaker, the grains tend to settle in the nodes (where there is no movement) reflecting the form of standing waves that are generated on the plate.

\subsection{Second stage: basic models to describe wave phenomena}

In this part, situations are proposed to formulate a mathematical model that represents the wave phenomenology presented and experienced in the previous stage. The mathematical description of the oscillatory motion is triggered by the experiments with the pendulum or the mass-spring system. Students explore and discover the relation between the motion (in particular position and velocity versus time) and the elementary trigonometric functions: sine or cosine, the basic "building blocks" of the mathematical wave model.

This stage is focused on the identification of the trigonometric functions describing the oscillations. No emphasis is put on the differential equations of the oscillating systems; students are guided directly to their solutions. Our goal is to define a minimum set of mathematical technicalities to encourage student understanding, avoiding unnecessary distractors. In this sense the mathematical description we are proposing may be considered a basic one; this approach will be followed throughout the work.

Once the relevance of the elementary trigonometric functions has been established, situations are proposed to investigate the role of the amplitude, frequency and phase parameters in the description of mechanical oscillations. The next step is the transition from oscillations to travelling mechanical waves. The challenge here is to describe the wave both in space and in time. To address this issue, situations are proposed that require a graphical representation of "snapshots" of the spatial extension of the wave, in order to identify an oscillatory dependence, both in space and time. Basically students have to explore what happens to an oscillation about an equilibrium point when it moves with a certain speed along a direction. A simple interactive application can be easily designed to encourage students' inquiry of this physical scenario and to lead them to discover for themselves the relation between the period $\mathrm{T}$ (or the frequency $f$ ) and the wavelength $\lambda$. This makes it possible to address the concepts of wavelength, frequency and propagation speed.

Quantisation occurring to waves trapped in a finite medium and interference are both classical experimental patterns having an analog in quantum mechanics. Both phenomena are strictly related to the superposition of travelling waves. Therefore, in order to introduce these two target topics, we need students to explore and become familiar with superposition, which is addressed both from a mathematical and a visual/geometrical point of view. It can be done by a simple experiment in which students listen to the superposition of two pure tones played with their smartphones (acoustic beats). This activity leads them to "visualise" both with their ears and their eyes what happens when you superpose two sine waves. Situations are also proposed in which students are asked to add waves both graphically or analytically (functions of only one variable, time or space, are considered at this stage). This requires a knowledge of the elementary algebra of trigonometric functions.

Interference of waves meeting at the same point from different paths and directions is a phenomenon that needs a lot of care from the educational point of view. We suggest to begin with a geometric and visual introduction, based on an interactive application we have specifically designed for this purpose, as shown Figure 2

With this application students can explore from a visual and geometrical standpoint what happens when two waves with the same wavelength meet at a point $\mathrm{P}$ on a screen. The waves start their travel from point 1

3D

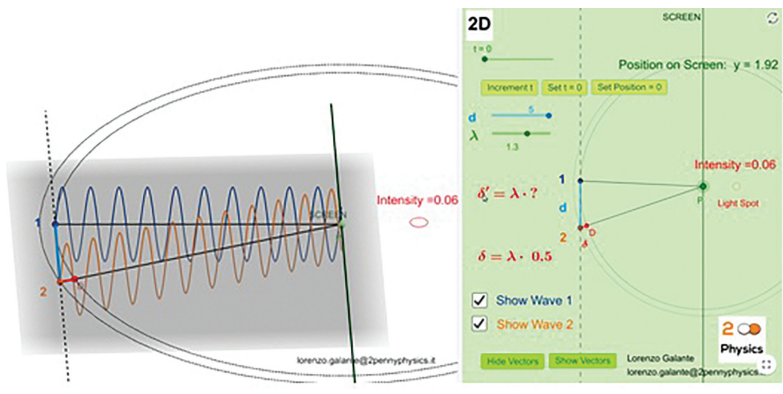

Figure 2: Simulation of interference and sum of vectors made with Geogebra. Source: 2-penny Physics (https://www.2pen nyphysics.it/2020/09/29/interferenza-luminosa/) 
and 2. Students have the opportunity to vary the distance $d$ separating points 1 and 2, thus varying the difference in the wave path length (red segment in the $2 \mathrm{D}$ part of the image). The application shows the physical scenario both in a two and a three dimensional environment and returns the intensity of the wave resulting from the superposition at point $\mathrm{P}$. The intensity is expressed both numerically and with a spot whose color intensity is proportional to the intensity of the resulting wave (in the image the difference in optical path is very close to half wavelength, the waves interfere in antiphase, as a consequence the resulting intensity is very close to zero). This framework will be very useful when the quantum experiment carried out by Davisson and Germer with electrons will be introduced.

\subsection{Third stage: atomic-scale phenomena}

In the fourth part, phenomena at the atomic level are presented. The kind of experiments shown are highly sophisticated and not accessible to the school laboratory. Therefore, references, videos or images of the real experiment, available through the internet, are used. We want to emphasize that the results of real experiments (rather than simulations) are shown in this stage. To characterize quantum systems, we selected two paradigmatic cases depicted in Figure 3 .

The left panel shows four snapshots of the detection screen from a "double-slit" experiment with electrons. In this experiment, electrons individually pass through a region with two "holes" before being detected on the screen. At first, a few electrons are detected (a) and (b) and their distribution on the screen appears to be random. But as time passes, which in this experiment is on the order of a few minutes, a pattern of alternating fringes begins to emerge as shown in (c) and (d), indicating that it is more likely to detect electrons in some places (light stripes) than others (dark stripes).

In the other experiment, shown in the right panel of Fig. 3. the hydrogen atom is shown "in vivo" [16]. The image on each panel is made up of many "snapshots" that detect the electron at different locations. The color grading indicates the places where the electron is most frequently or likely to be detected (red) to where it is least likely to be detected (blue). In addition, each subpanel indicates different "states" of the atom that are accessed by increasingly exciting it from (a) to (d) with laser light.

We would like to highlight that, as in the first stage, the inquiry here points to phenomenological aspects. The situations raised seek that the student identifies the central elements of the experiment, in which physical quantities are measured. Here it is key to emphasize the presence of a common "pattern": a distribution that goes from places where the electron is preferentially found to others where it is almost never detected. This aspect is manifested both in the detection screen of the electron double slit experiment and in the "snapshots" of the electron in the hydrogen atom.

\subsection{Fourth stage: basic aspects of wave quantum mechanics}

In the last part, we set out to analyze the extent to which previous quantum experiments support a description in wave terms. In this sense, the possible analogies between quantum phenomena and mechanical waves are fundamental. Each quantum phenomenon presented has a mechanical analog, which can be used didactically as an anchor for the concept, although the character of the "wave" is different.

The central idea is the existence of common wave mathematics that is at the base of completely different physical models: the macroscopic wave physics accessible to our everyday experience (waves in water and their interference, normal modes in a string or in a pipe, etc.) the wave model for atomic or subatomic physics, not accessible to direct and everyday experience. The quantum experiments presented in the previous section reflect two fundamental aspects, the statistical nature of the phenomena, which suggests a description in terms of probability distributions, and the inherent wave character, since these distributions (experimental results) admit a direct description in wave terms, i.e.
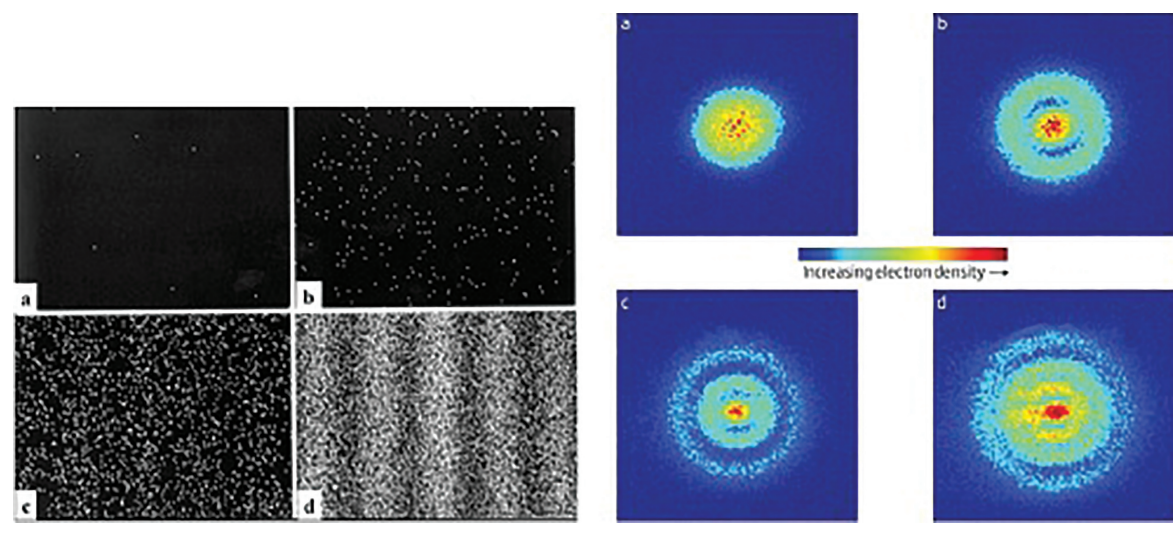

Figure 3: (Left): Electron interference. (Right): "Photographs" of the hydrogen atom. 
electron diffraction and interference, stationary states of the atom, tunnel effect, etc.

Regarding the electron double-slit experiment note that, although the phenomenon of interference and electron diffraction was put into evidence from the first days of quantum mechanics, for instance in electron diffraction by a crystal experiments, the double-slit version presented here has additional didactic value as it allows to be more easily connected to its classical water wave interference counterpart of Fig. 1(a). The situations in the third stage promote this analogy.

Similarly, in the hydrogen atom experiment outlined in Fig. 3(right), the four sub-panels reflect different patterns of standing waves of probability distributions, which are accessed by exciting the electron to higher energy levels. These patterns have mathematical aspects analogous to those that describe the standing wave patterns in a string, or more appropriately the circular plate of Figures 1.b) and (c), respectively. Here again, the situations raised will promote a connection between both phenomenologies.

Finally, the analogy is explored in the case of the tunnel effect (not presented here), whose introduction in the first stage in a classical context gives it a general character, not only associated with quantum effects, as the subject is usually presented.

In all the cases analyzed, the emphasis will be on exploring via analogy the probabilistic nature of the quantum wave model, which is naturally connected with the statistical character of the quantum experiments presented in the previous stage.

The wave description emerges from the phenomenology presented. But it has a particular character. First, there is an inherent statistical character. Given similar equivalent conditions the quantum system evolves to different final stages. This is in contrast to classical systems where the same initial state generates the same final state. This statistical character is evident both in the experiment of the double slit and the one of the location of the electron in different "places" around the nucleus. Despite the apparent "randomness" what is observed is that with enough statistics, patterns begin to emerge. These "probability" patterns of detecting, for example the electron in a given place can be described in terms of ordinary wave effects, like interference, standing waves and so on.

The quantum object is not associated directly to a wave, but rather in a rather indirect and subtle way, through its statistical behavior, which finds its mathematical description in terms of waves and probability.

\section{The didactic use of These Analogies}

In this work we consider the following categorization of the analogies proposed by Curtis and Reigeluth [17] about analogies present in textbooks. Although the application context is different, we take it because it is useful to analyze the didactic proposal to teach with quantum concepts:

- Nature of base domain: In this case, it refers to mechanical waves: in a wave bucket, a string fixed at one end, and salt distribution in a metal plate attached to a speaker.

- Analogical relationship: It is Structural - Functional. In both contexts there are spatial distributions with defined patterns associated with different physical models. These can be described through a common mathematical model of wave superposition. In the macroscopic context there is a range from maximum to minimum concentration of water on the surface, salt on the plate, or separation of strings from the equilibrium point etc. This corresponds, in the quantum context, to places where the electron is more likely to be detected than others, for example in the light and dark fringes of the interference pattern of the double-slit experiment. The same is true for the normal mode probability distribution of the electron in the hydrogen atom.

- Condition: Concrete/Abstract. The nature of the base domain is concrete and the nature of the target domain is abstract. The base domain refers to familiar aspects of mechanical waves: water on a bucket, salt in a plate that receives vibration. However, the target domain is abstract in the sense that both the electron interference pattern or the "snapshots" of the hydrogen atom are far from everyday experience.

- Level of enrichment: The enriched analogy contains the limitations of the analogical relationship: In this case, the quantum system intrinsically possesses the concept of probability, which is absent in the classical context analyzed. In the classical system, particles and waves are totally separate entities. In the quantum framework, from the wave perspective that we address, the particle is an emergent (as a wave packet).

- Position: Advance organizer: The analogy is presented to the students like an introduction of the new model that will be developed during the teaching activity.

\section{Conclusions}

In this work, we have outlined a structure of a didactic sequence based on experimental results and analogies between classical and quantum experiments to teach basic aspects of quantum mechanics in the last year of high school. Our approach does not place the waveparticle duality on an equal footing, but rather favors the wave aspects in a true wave quantum mechanics. The approach is devoid of the paradoxes and intellectual challenge that comes with imagining that something is both a wave and a particle, being entities so disparate 
in our daily experience. In our opinion, this has several advantages. On the one hand, the phenomenology of mechanical waves provides an intuitive framework that invites to develop a mathematical model based on waves, which can then be applied by TWA methodology to quantum phenomena.

On the other hand, although they are not explored in this work, there are two fundamental aspects of quantum mechanics that arise naturally when considering the starting point of waves: the Heisenberg uncertainty principle and the emergence of a particle as a package. or sum of waves. For the first, we have already outlined a proposal that allows addressing the connection between dispersions in position and momentum in quantum systems in a way analogous to the dispersions in sound intensity distributions when represented as a function of frequency and time [7.

To conclude, we list the basic knowledge of mathematics and physics necessary to support the analogy classical-quantum wave phenomena.

- Basic trigonometry

- Maxima and minima of a mathematical function

- Wave interference

- Basic probability and statistics

- probability distribution of an observable,

where the main target quantum concepts are:

- Quantum probability distribution

- Electron interference

- Discrete energy levels in the atom

- Standing wave electron probability distribution

The design of a sequence of questions and problems for students based on the approach presented here is under development, and its subsequent implementation with high school students will allow evaluating its viability.

\section{References}

[1] K. Krijtenburg-Lewerissa, H.J. Pol, A. Brinkman and W.R. van Joolingen, Physical Review Physics Education Research 13, 010109 (2017).

[2] E. Cataloglu and R.W. Robinett, American Journal of Physics 70, 238 (2002).

[3] A. Kohnle, I. Bozhinova, D. Browne, M. Everitt, A. Fomins, P. Kok, G. Kulaitis, M. Prokopas, D. Raine and E. Swinbank, European Journal of Physics 35, 015001 (2014).

[4] I.D. Johnston, K. Crawford and P.R. Fletcher, International Journal of Science Education 20, 427 (1998).

[5] S.B. McKagan, K.K. Perkins and C.E. Wieman, Physical Review Physics Education Research 4, 010103 (2008).

[6] C. Baily and N.D. Finkelstein, Physical Review Physics Education Research 6, 010101 (2010).

[7] L. Galante, M. Arlego, M. Fanaro and I. Gnesi, Physics Education 54, 015017 (2018).

[8] R.K. Coll, B. France and I. Taylor, International Journal of Science Education 27, 183 (2005).
[9] I. Halloun, Science \& Education 16, 653 (2007).

[10] D.P. Portides, Science \& Education 16, 699 (2007).

[11] A. Archer, M. Arcâ and N. Sanmartí, Science Education 9, 398 (2007).

[12] R. Duit, Science Education 75, 671 (1991).

[13] S.M. Glynn, in: The psychology of learning science (Hillsdale, New Jersey, 1991).

[14] A.G. Harrison and D. Treagust, in: Using analogies in middle and secondary classrooms (Corwin Press, Thousand Oaks, 2008).

[15] A.S. Stodolna, A. Rouzée, F. Lépine, S. Cohen, F. Robicheaux, A. Gijsbertsen, J.H. Jungmann, C. Bordas and M.J.J. Vrakking, Physical Review Letters 110, 213001 (2013).

[16] https://www.youtube.com/watch?v=jvO0P5-SMxk

[17] V. Curtis and C.M. Reigeluth, Instructional Science 13, 99 (1984). 